\title{
Déformations cycliques et irréversibles dans les remblais argileux
}

\section{MIEUSSENS}

Laboratoire régional des ponts et chaussées de Toulouse

Complexe scientifique de Rangueil

1, avenue du Colonel-Roche 31400 Toulouse
L'étude du comportement des sols compactés mis en remblai est abordée à partir des enseignements que l'on peut tirer des mesures de déformations. Les observations faites sur un grand remblai expérimental ont permis de mettre en évidence l'importance du phénomène classique de reptation des pentes. L'enregistrement des mouvements verticaux confirme que des cycles de gonflement et retrait sont bien à l'origine de ces déplacements. Par contre, sur ce même site et sur d'autres ouvrages, il est démontré que des phénomènes de type fluage pourraient expliquer certains déplacements horizontaux et verticaux, généralement localisés en profondeur et pour lesquels il n'existe pas de méthode de prévision dans le cas particulier des sols non saturés.

\section{Irreversible and cyclic strains in clay embankment}

The behaviour of compacted soils used in embankment is enlighted by the results of deformation measurement. The data collected on an important experimental embankment show the importance of the crawling of slopes. The recording of vertical movements shows that cycles of swelling and shrinkage are the source of this deplacements. On the other hand, on this same site and for other works, it has been shown that fluage behaviour may explain some deplacements verticaly and horizontaly, usually located in depth and for thern it doesn't exist any method of predicting specially for unsaturated soils. 


\section{Introduction}

Les remblais argileux sont fréquemment le siège de déformations verticales et horizontales, se traduisant par des affaissements plus ou moins localisés et par des fissures souvent longitudinales. Ces désordres, généralement mineurs, nécessitent un entretien fréquent, le colmatage des fissures sur la chaussée et la reprise périodique du profil en long. Dans certains cas, cette pathologie peut prendre une ampleur inacceptable, les affaissements représentent un risque pour l'usager et l'évolution de la fissuration peut faire craindre un risque de rupture du remblai ou du moins la formation d'un décrochement sur la voie.

Ces phénomènes peuvent apparaitre rapidement après la fin de construction ou bien se manifester sur des ouvrages très anciens. Selon les sites, l'évolution peut sembler régulière ou au contraire se faire par crise, avec des corrélations apparentes avec des périodes de sécheresse ou au contraire de forte pluviométrie.

Il semble important de rappeler que le comportement des corps de remblai appartient à l'un des domaines le plus mal connu de l'ingénieur, à savoir celui des sols non saturés. Par ailleurs, le dimensionnement d'un ouvrage se fait le plus souvent par rapport à la rupture et les calculs en déformations sont exceptionnels, alors que les problèmes posés par les corps de remblais sont le plus souvent liés aux déformations. Il existe quelques outils de calculs en éléments finis, difficiles à utiliser, d'autant que les paramètres représentatifs d'un sol compacté non saturé sont eux-mêmes difficiles à mesurer.

Depuis les travaux anciens de Proctor, d'importants efforts de recherche ont été réalisés, mais ils ont été essentiellement orientés vers une amélioration des techniques de mise en œuvre. En France, les Recommandations pour les terrassements routiers, éditées entre janvier 1976 et octobre 1981 (LCPC-SETRA), avaient déjà introduit la notion « d'état » du matériau, en particulier lié à la teneur en eau, ainsi qu'une classification prenant en compte des mélanges granulométriques de fines et de gros éléments. Plus récemment, le Guide technique pour la réalisation des remblais et couches de forme (LCPC-SETRA, septembre 1992) propose des méthodes d'études des matériaux en fonction des risques d'évolution (classe R qui concerne les matériaux rocheux évolutifs).

Cette notion de roche évolutive ne se limite pas à un changement granulométrique dû à l'extraction et au compactage, mais parfois aussi à une dégradation progressive de ses caractéristiques mécaniques par imbibition. Cette notion d'évolution est fondamentale car elle suppose implicitement qu'un matériau compacté, mis en œuvre correctement en suivant les règles de l'art, est susceptible d'évoluer dans le temps. Les progrès dans ce sens sont encore timides, et il existe peu ou pas d'essais de routine permettant de prévoir le comportement à long terme d'un corps de remblai, tassements et déformations horizontales, sous le simple effet des variations de teneur en eau. Les essais tels que la fragmentabilité et la dégradabilité ne sont que des indicateurs de risques.

Le principal objet de cet article est de présenter des exemples de mesures de déformations faites sur des remblais routiers et autoroutiers, mesures qui pourront servir de base de réflexion pour expliquer les mécanismes physiques probables et tenter d'expliquer le comportement complexe des sols compactés mis en remblai. On pourra ainsi mieux orienter la conception. l'étude des ouvrages et leur réparation éventuelle.

\section{2}

\section{Enseignements tirés des mesures sur le remblai expérimental de La Jorle}

Le remblai de La Jorle a été construit entre 1979 et 1981 ; il a fait l'objet d'une importante instrumentation, certains appareils sont encore suivis à ce jour et une instrumentation complémentaire a été mise en place en 1996. Il s'agit d'un grand remblai construit avec des marnes argileuses, d'une hauteur de 30 mètres et qui permet le franchissement par l'autoroute A62 du petit ruisseau de La Jorle. On ne présentera ici que les résultats sur les déformations cycliques. Des informations plus complètes se trouvent dans l'article de D. Thouret et al. (1992), ainsi que dans les rapports périodiques de mesures établis dans le cadre d'une recherche associant le Laboratoire régional des ponts et chaussées de Toulouse, la société des Autoroutes du Sud de la France et Scetauroute. Les principaux résultats de cette recherche ont été présentés par C. Mieussens (1986) et (1997), ainsi que dans la thèse de J. Camapum à l'INSA de Toulouse en 1985.

La figure 1 donne la position schématique d'une partie de l'instrumentation dont les résultats des mesures seront analysés dans le présent article.

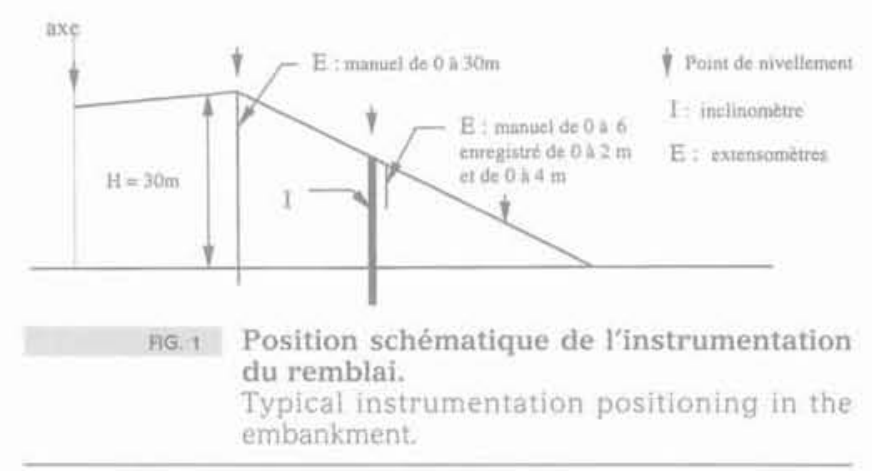

\section{Tassements à long terme}

La figure 2 donne un exemple des résultats des mesures de tassements faites sur ce remblai, par nivellement. Elles correspondent aux seules déformations différées, l'origine étant prise un an environ après la fin de construction.

Plusieurs observations intéressantes peuvent être faites sur ces courbes qui correspondent à plus de seize années de mesures :

- le tassement dans l'axe de la voie est sensiblement plus faible que celui mesuré en crête de talus, ce qui 


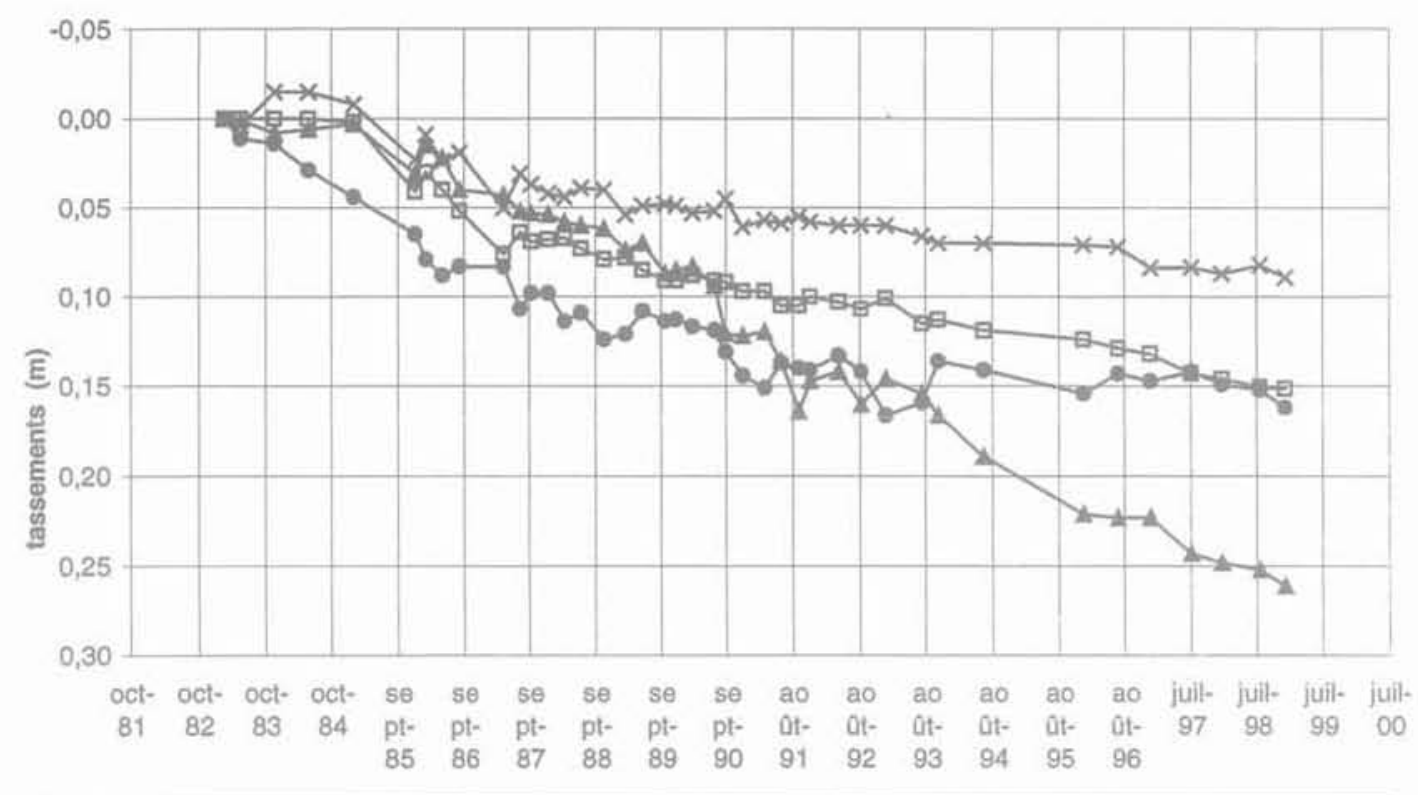

$\rightarrow$ axe vole $\quad \rightarrow$ crête talus $\quad \rightarrow$ mi-talus $\quad \rightarrow$ tiers inférieur talus

FIG. 8 Tassements différés en différents points d'un remblai de 30 mètres de haut (origine des mesures : un an après la fin de construction).

Long term settlements in various location in a 30 meter high embankment forigin ; on year after the construction completion).

confirme la tendance d'un basculement des crêtes de talus par rapport à la plate-forme ;

- le «tassement $)$ le plus important est mesuré à mitalus ; il est pratiquement linéaire en fonction du temps depuis 1984;

- les faibles tassements et les soulèvements observés entre 1983 et 1984 s'expliquent par des phénomènes de gonflement par imbibition, bien confirmés par des mesures d'extensométrie avec des tassomètres multipoints (Figs. 3 à 5 ).
Cet ensemble de mesures montre que le seul nivellement n'est pas suffisant pour expliquer le comportement du corps de remblai, le gonflement peut masquer temporairement un tassement et le retrait donner une impression d'accélération. Par ailleurs, les « tassements » plus importants en crête et surtout à mi-talus s'expliquent en réalité par une combinaison d'un tassement et d'un déplacement horizontal qui sera examiné plus loin.

La figure 4 montre que, dans le cas du remblai expérimental de la Jorle, les cycles de gonflement - retrait

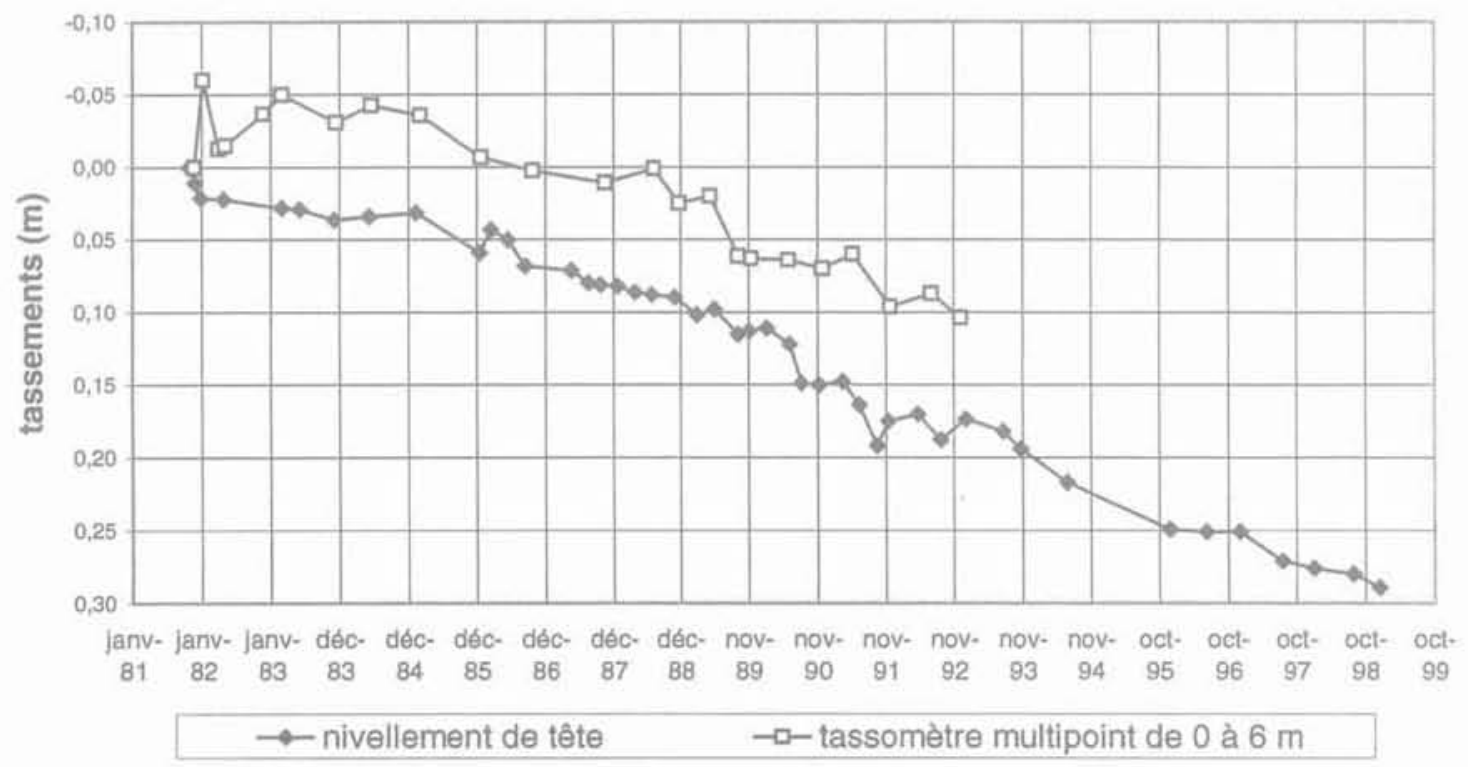

FiG. 3 Comparaison des déformations verticales mesurées par topographie et par extensométrie entre la surface du talus et une référence à -6 mètres de profondeur - mesures à mi-talus d'un remblai de 30 mètres de hauteur.

Comparison between vertical movements measured by surveving and extensometer located between the slope surface and six meters deeper at mid slope of a 30 meters high embankment. 


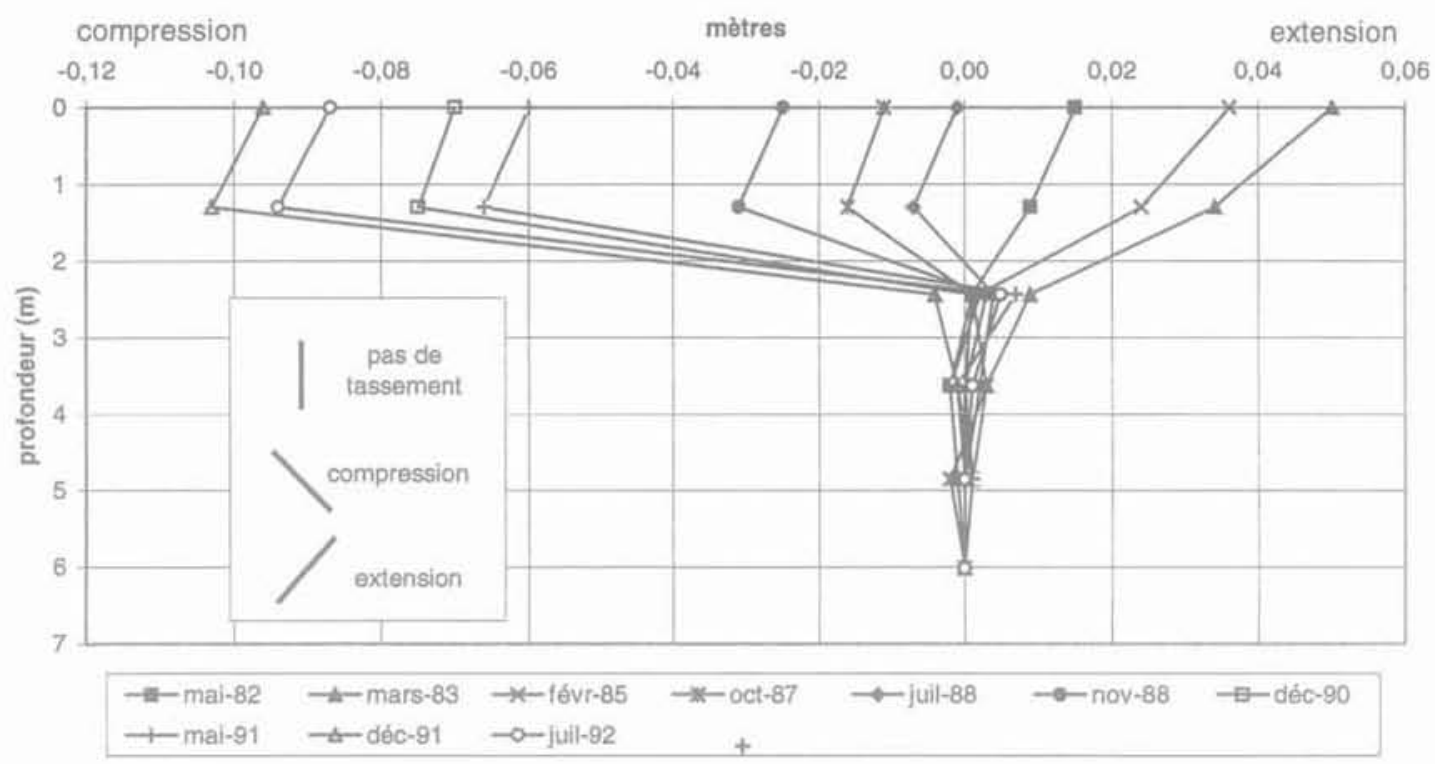

Ho.4 Mesures des compressions - extensions de 0 à 6 mètres de profondeur (remblai expérimental de la Jorle). Compression and extension measurements (0-6 meters depth at la Jorle experiment site).

concernent, à mi-talus, essentiellement la couche de surface de 0 à $2 \mathrm{~m}$ de profondeur. Après une période de gonflement dont le maximum se situe en mars 1983, on observe une tendance irréversible à la compression. La couche de surface parait conserver une épaisseur constante, il s'agit plus probablement d'une mesure non représentative due à une mauvaise liaison entre le repère situé à - 1,2 mètre de profondeur et le terrain. Un nouveau système a été mis en place en 1996.
La figure 5 donne les résultats des mesures de tassement relatif en fonction du temps et de la profondeur sous la crête de talus. Le point fixe de référence correspond à la base du remblai. L'incertitude des mesures est probablement assez grande. On peut toutefois noter que la topographie de surface (Fig. 2) confirme les ordres de grandeur pour la période considérée, soit un tassement de l'ordre de $10 \mathrm{~cm}$ en 10 ans.

Tassements cumulés en fonction de la profondeur (crête du talus)

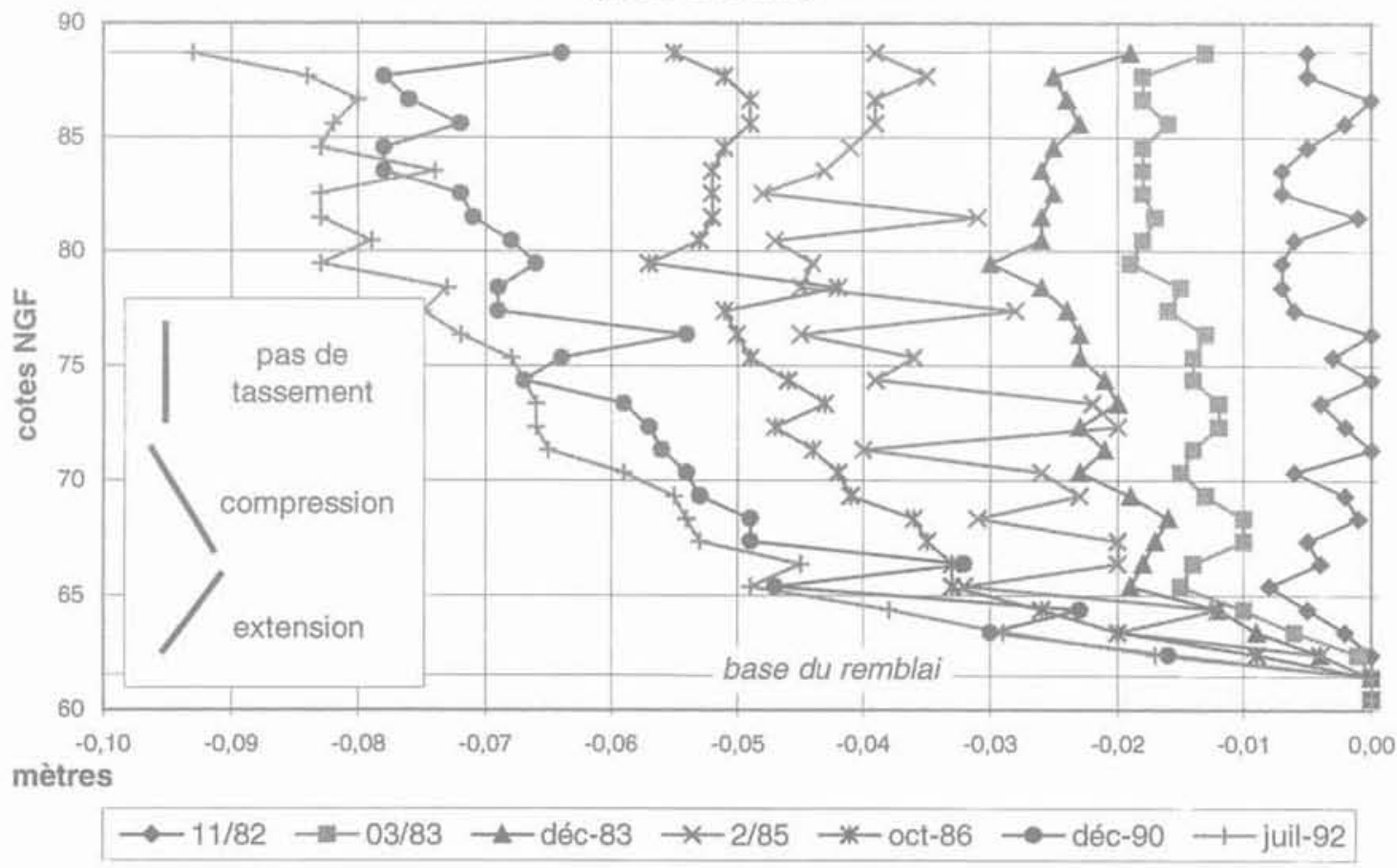

FIG.5 Mesures des tassements et gonflements cumulés en crête du talus du remblai. Cumulated settlement and swelling measured at slope crest 
Les courbes de tassements cumulés en fonction de la profondeur (Fig. 5) mettent en évidence les tendances suivantes:

- les 2 à 3 mètres supérieurs de remblais (cotes 89 à 86) sont soumis à des cycles de gonflement et retrait, qui correspondent assez bien aux cycles saisonniers ;

- entre 2 et 10 mètres de profondeur (cotes 86 à 80 ), la variation d'épaisseur de la couche est négligeable : - entre 10 et 25 mètres de profondeur (cotes 80 à 65), les tassements augmentent progressivement en fonction du temps:

- les tassements les plus importants concernent la couche de base sur 4 mètres d'épaisseur entre les cotes 65 et 61 NGF ( $50 \%$ du tassement total en 1992), avec une évolution également progressive.

Depuis 1992, les mesures avec le tassomètre multipoint de profondeur sont devenues impossibles, probablement à cause de la déformation latérale du tube. La précision était également devenue insuffisante par rapport aux phénomènes mesurés. En 1996, un nouveau système de mesure par extensométrie a été mis en place à mi-talus. La figure 6 décrit le principe de ce matériel, avec un capteur relié à une centrale d'enregistrement.

La figure 7 donne les résultats des mesures respectivement de 0 à $2 \mathrm{~m}$ et de 2 à $4 \mathrm{~m}$ de profondeur (par différence avec une mesure de 0 à $4 \mathrm{~m}$ ).

Ces mesures plus précises qu'avec le tassomètre multipoint confirment les variations cycliques annuelles des déformations, avec une amplitude de 30 à $35 \mathrm{~mm}$. ainsi que leur aspect non réversible. On notera que la non-réversibilité est actuellement du même ordre de grandeur entre 0 et 2 mètres et entre 2 et 4 mètres de profondeur, ce qui pourrait traduire un approfondissement des phénomènes.

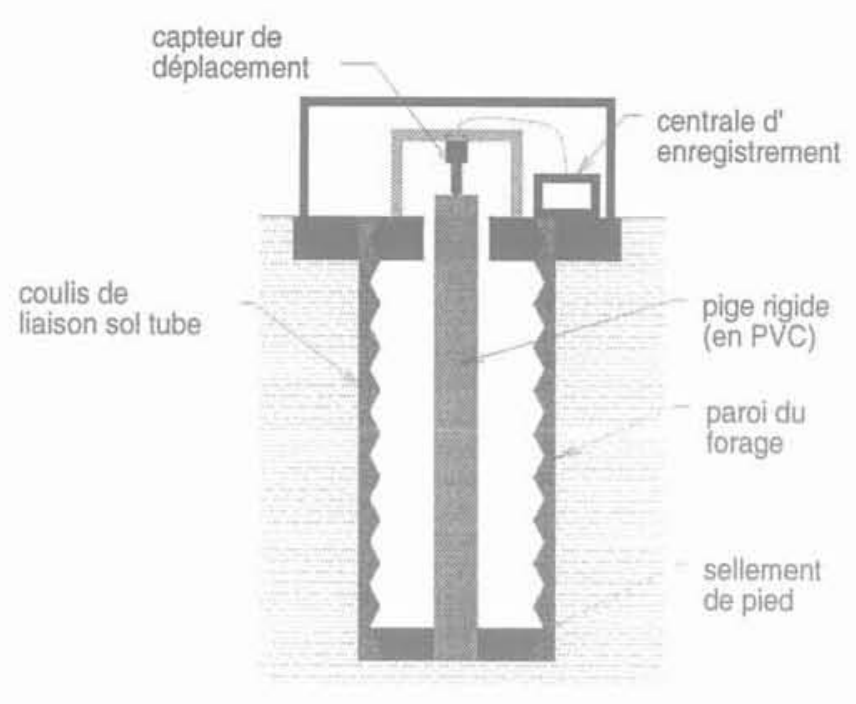

HG.6 Principe de la mesure par extensométrie dans les talus de remblais.

Extensometer measurement in the slope of embankment.

\section{2}

\section{Déplacements horizontaux}

Les mesures inclinométriques sont à la base des principales informations sur les déplacements horizontaux. La figure 8 donne les résultats des mesures à mitalus pour le remblai de la Jorle (implantation précisée sur la figure 1). Le tube inclinométrique a été changé trois fois à la suite d'un ( cisaillement $»$ au niveau des premières couches de remblai (13 à 14 mètres de pro-

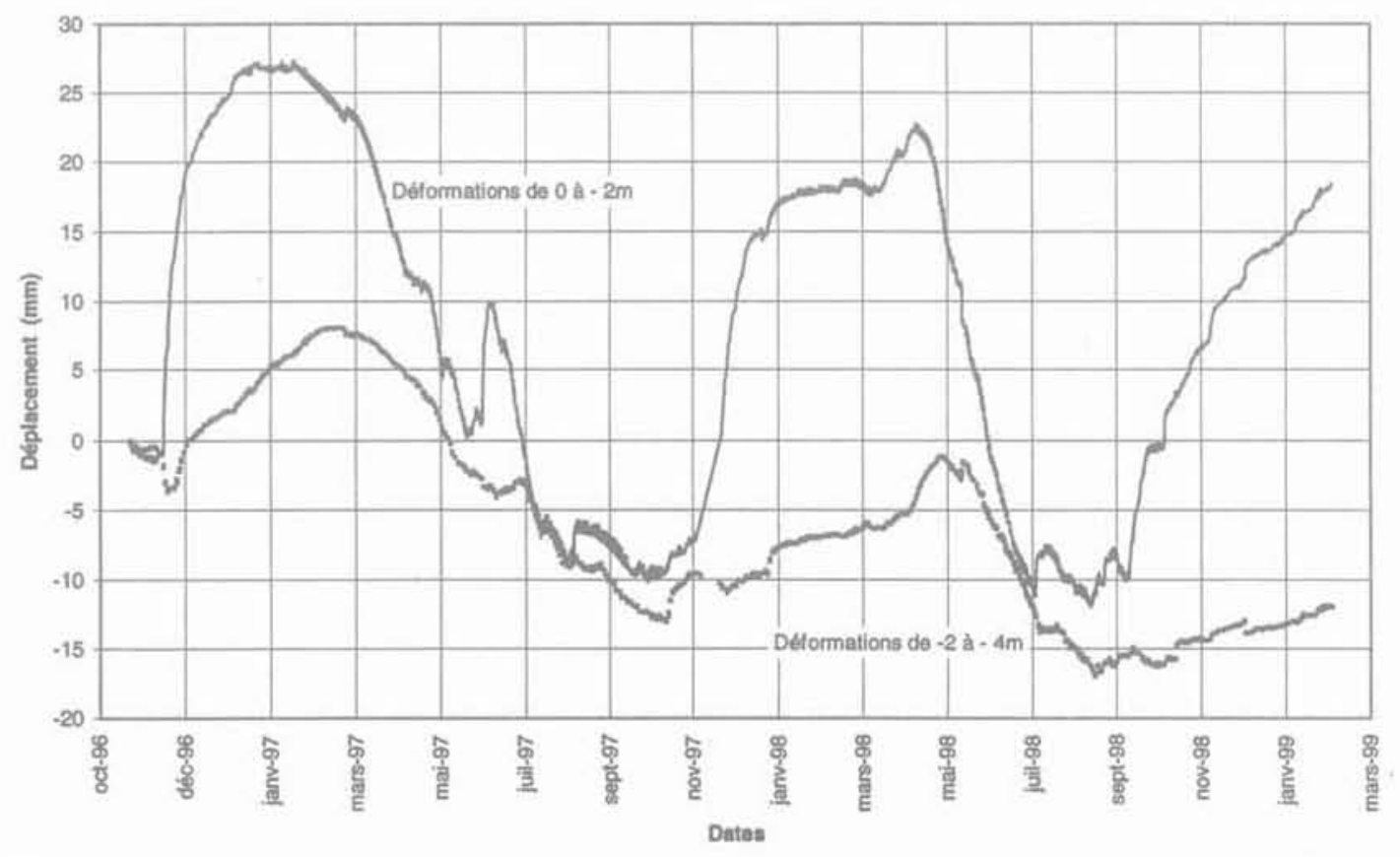

F16. 7 Variations cycliques d'épaisseur entre 0 et 4 mètres de profondeur. Thickness variations between 0 and 4 meter depth. 


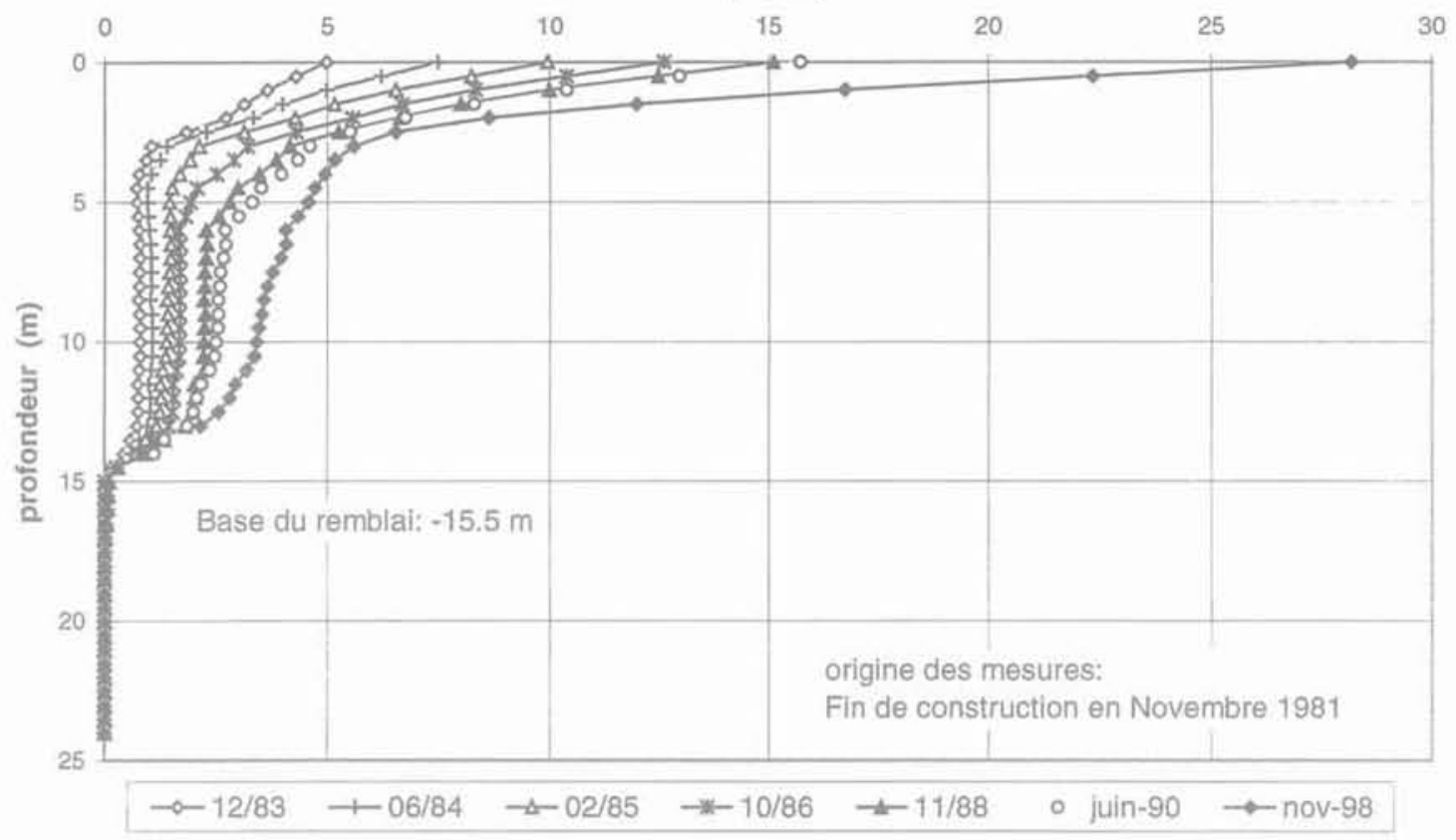

FG. 8 Mesures inclinométriques à mi-talus du remblai de La Jorle (extraits). Inclinometer measurement at mid slope (La Jorle experiment site).

fondeur). Cette déformation reste localisée, puisque les mesures inclinométriques plus à l'aval, dans le talus, ne traduisent pas une continuité des déplacements. Il n'y a pas non plus de flambage du tube, qui serait caractérisé par une certaine symétrie des rotations, Cette déformation correspond à la couche de remblai où les tassements sont les plus importants ( 1 à $2 \%$ d'après la figure 5), une plastification locale du matériau n'est donc pas à exclure, La figure 9 montre que l'évolution de cette déformation locale, traduite en rotation, est pratiquement linéaire en fonction du temps.

La figure 8 montre également que les déformations les plus importantes concernent la couche de surface du talus, avec un approfondissement progressif en fonction du temps. Depuis 1990, la déformation concerne l'ensemble du remblai, mais dans des proportions assez faibles à partir de 6 mètres de profondeur.

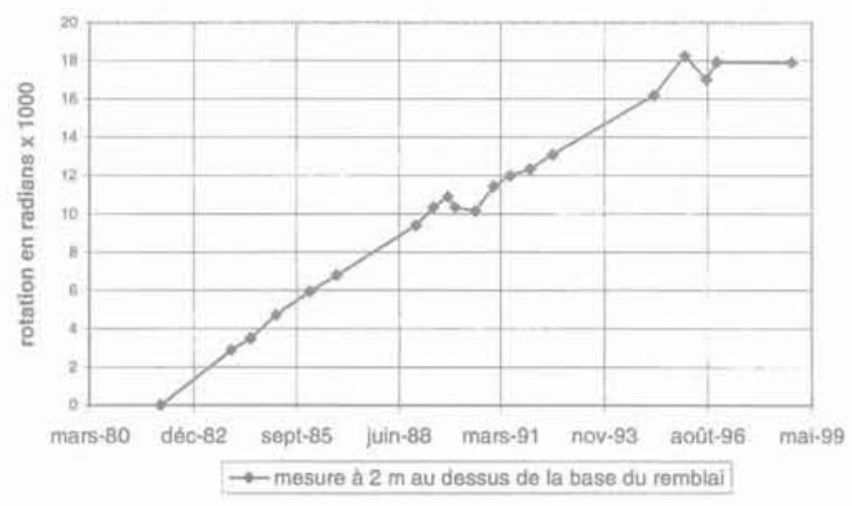

FIG. 9 Evolution des rotations à 2 mètres audessus de la base du remblai en fonction du temps.

Rotation evolution versus time 2 meters above the embankment base.

\section{3}

\section{Trajectoire d'un point à mi-talus}

La combinaison des mesures des déformations verticales et des déplacements horizontaux sur une même verticale permet de donner des indications sur les mouvements absolus et relatifs d'un point du talus du remblai.

La figure 10 donne cette information dans le cas d'un point situé à mi-talus. La trajectoire absolue correspond à la combinaison des mesures de nivellement et d'inclinométrie par rapport à un point d'ancrage fixe. Les mesures relatives sont interprétées par rapport à un point supposé fixe à 6 mètres de profondeur sous la surface du talus fle déplacement horizontal mesuré à 6 mètres de profondeur n'a pas été pris en compte sur ce graphique).

Cet ensemble de mesures permet d'expliquer les importants mouvements observés à mi-talus par le phénomène de reptation de pente. Comme l'indique la figure 11, en phase d'humidification, le sol compacté a tendance à gonfler, avec une déformation normale à la surface du talus.

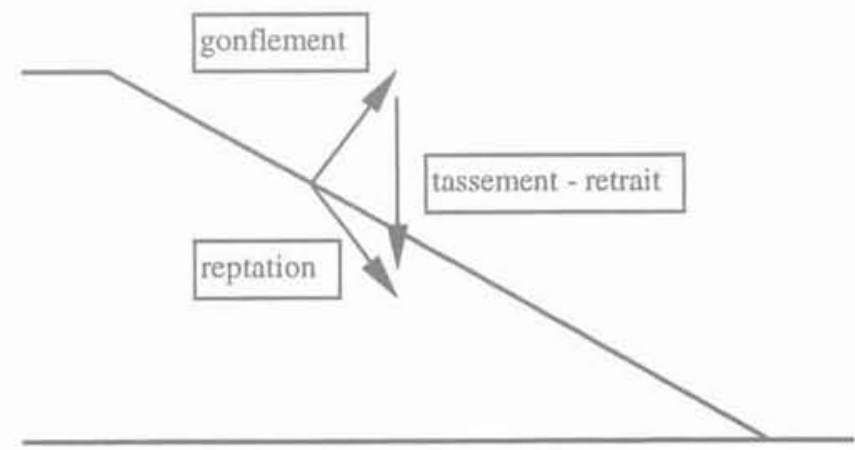

FG.11 Schéma de principe du phénomène de reptation de pente.

Slope crawling schematic principle. 


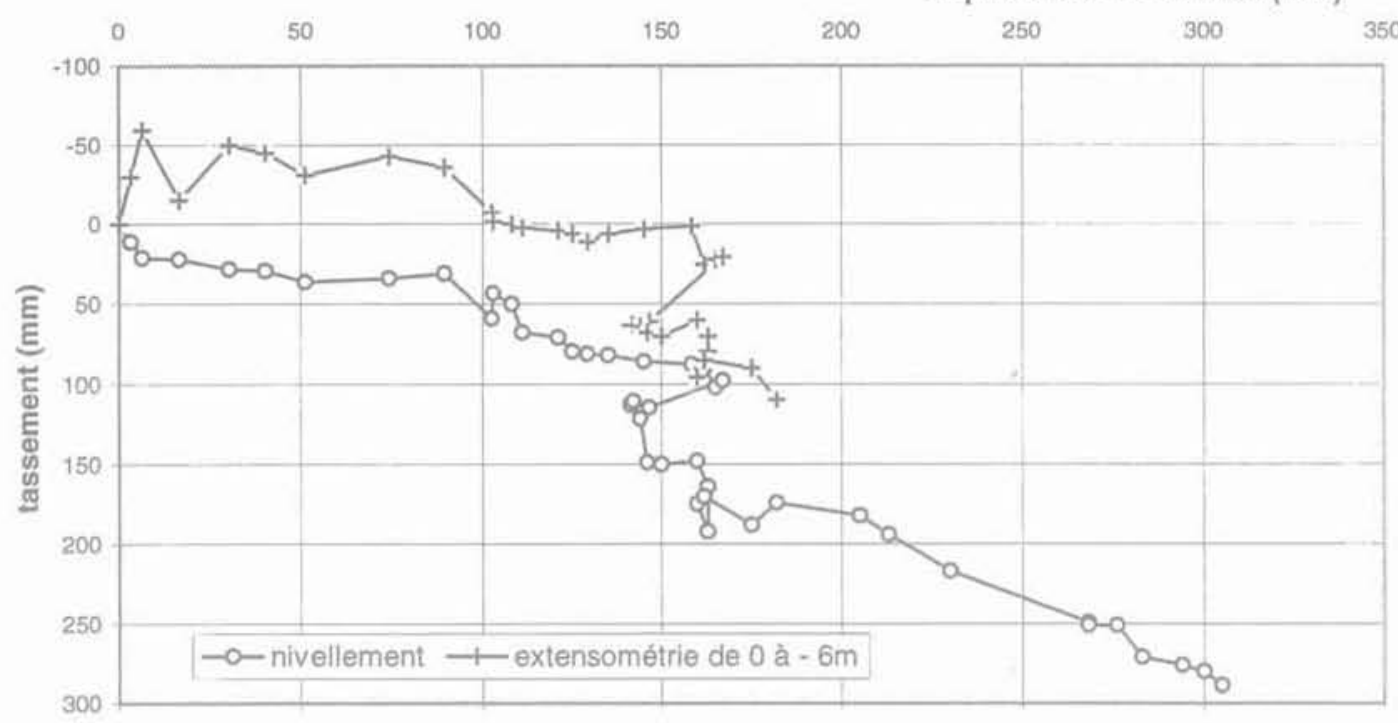

(a) - Trajectoire d'un point à mi-talus (Novembre1981 à Décembre 1998)

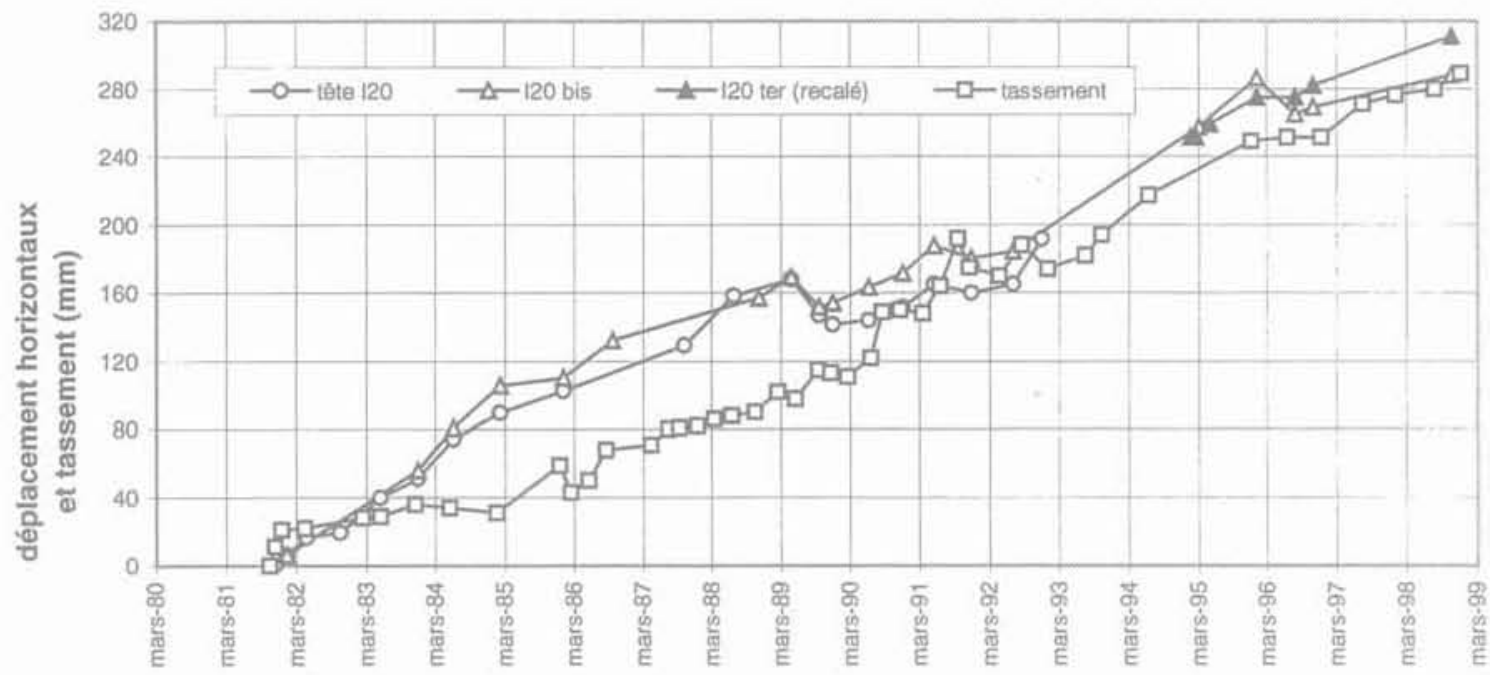

FiG. 10 Trajectoire d'un point situé à mi-talus du remblai définie par combinaison des mesures inclinométriques et des mesures de tassement par extensométrie ou nivellement.

Path of a point located at mid slope determined using settlement measurement extensometer reading, surveying and inclinometer measurement.

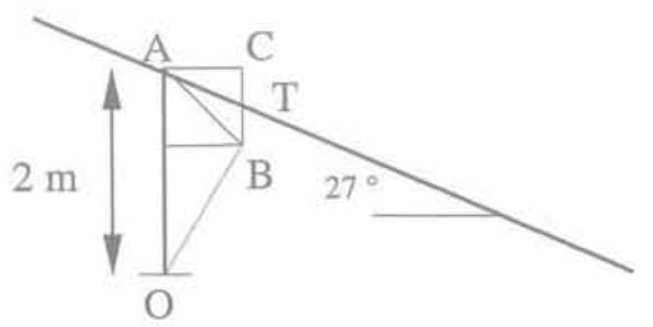

FG. 12 Incidence de la reptation sur les mesures. Impact of slope crawling on the measurement.
Comme le montrent les figures 4,6 et 7 , la reptation concerne essentiellement la couche de surface du talus qui est la plus exposée aux cycles climatiques. Les tassements mesurés par topographie sont donc affectés par cette composante du déplacement, qui est orientée en moyenne, pour ce remblai, à 45 degrés. Une simple analyse géométrique (Fig. 12) montre également que la mesure par extensométrie des variations d'épaisseur d'une couche doit tenir compte du déplacement de la tête par reptation.

Un calcul schématique donne le résultat suivant:

Si le point $\mathrm{O}$ est fixe et $\mathrm{AC}=\mathrm{CB}=0,3 \mathrm{~m}$, l'extensomètre de longueur initiale $\mathrm{AO}=2 \mathrm{~m}$ aura une longueur finale telle que $\mathrm{OB}=1,73 \mathrm{~m}$. La réduction réelle d'épaisseur de la couche est égale à TB $=0,147$ $\mathrm{m}$ et non pas $C B(0,3 \mathrm{~m}$ d'après la topographie) ni $\mathrm{OA}-\mathrm{OB}=27 \mathrm{~cm}$ d'après l'extensomètre. 


\section{3}

\section{Deux autres exemples de mesures de déformation de remblais routiers}

La figure 13 donne un exemple de corps de remblai instrumenté de façon classique avec un tube inclinométrique, mais aussi avec des chaînes extensomé- triques, verticales, subhorizontales et inclinées à 30 degrés. Il s'agit d'un remblai de 25 mètres de haut, également édifié avec des argiles marneuses, mais dont la partie supérieure de 0 à 8 mètres de profondeur a des caractéristiques mécaniques particulièrement faibles.

Sur une période relativement courte, les mesures inclinométriques ont mis en évidence une zone de rotations locales importantes, conduisant finalement à un cisaillement du tube.

axe voie

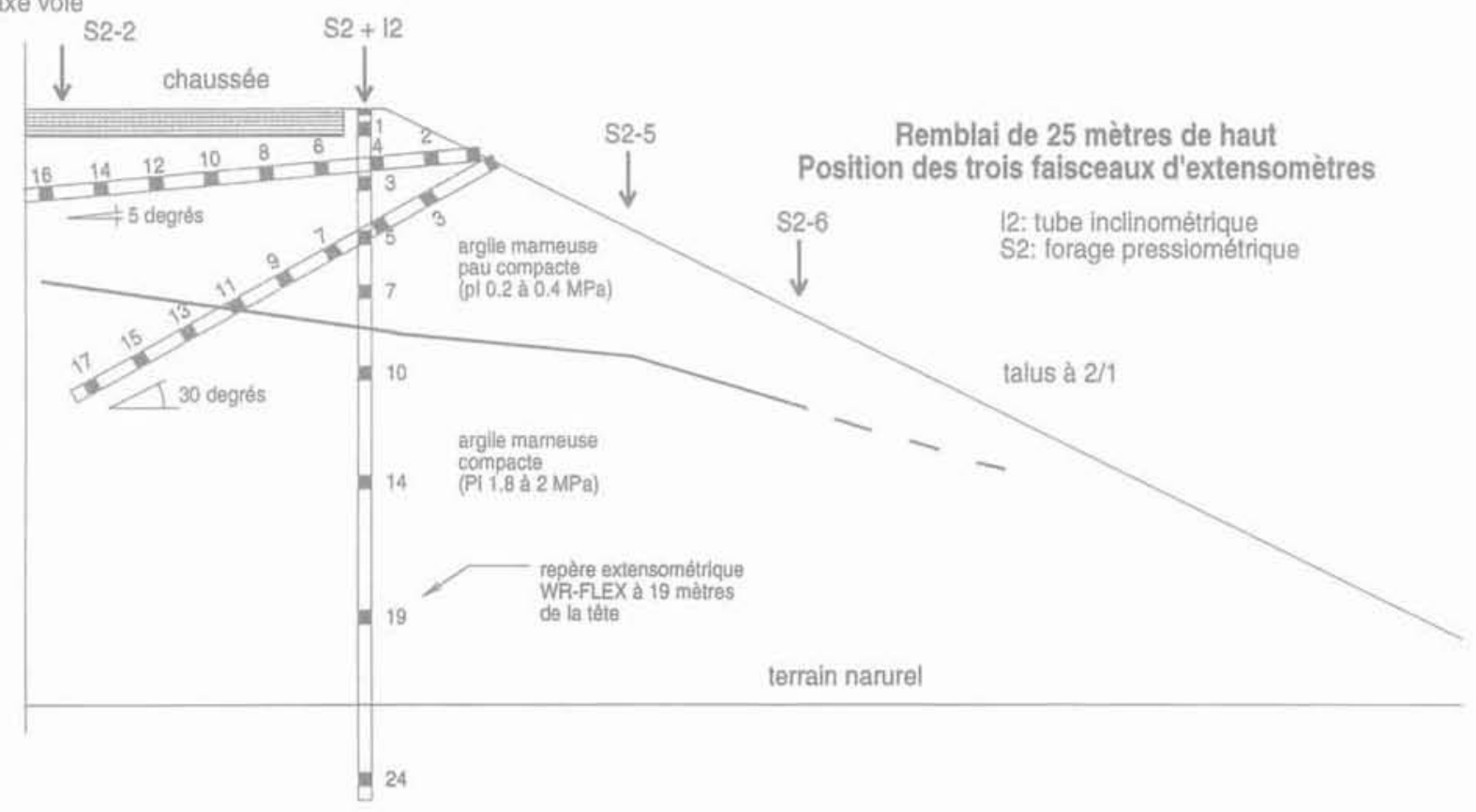

FG, 13 Profil en travers et instrumentation du remblai. Side view and instrumentation in the embankment.

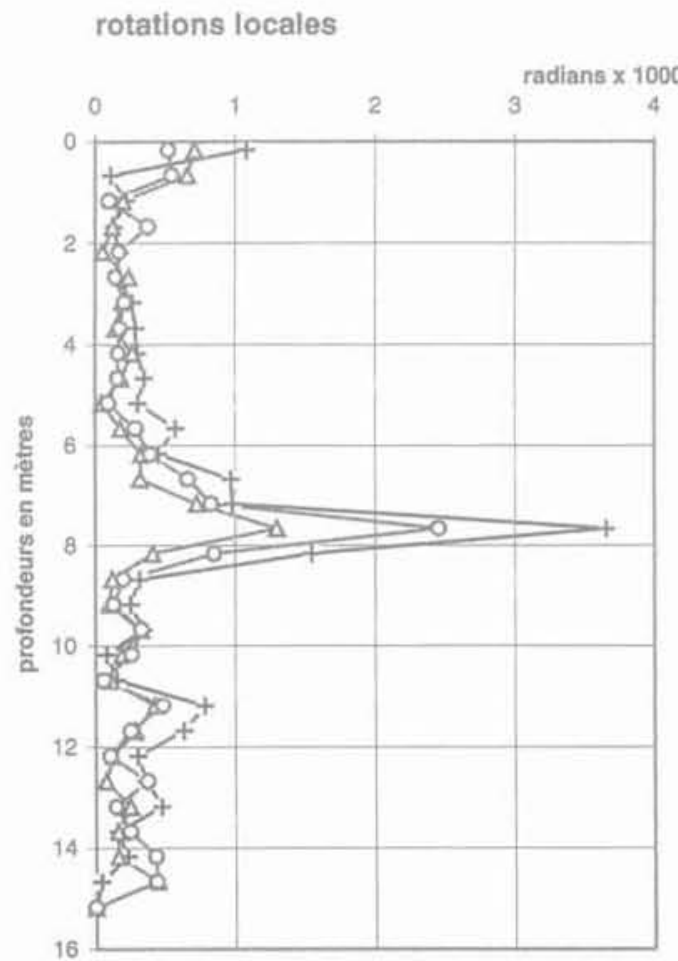

$+06 / 09 / 90-\Delta-06 / 03 / 90-0-08 / 06 / 90$

\section{déplacement cumulés}

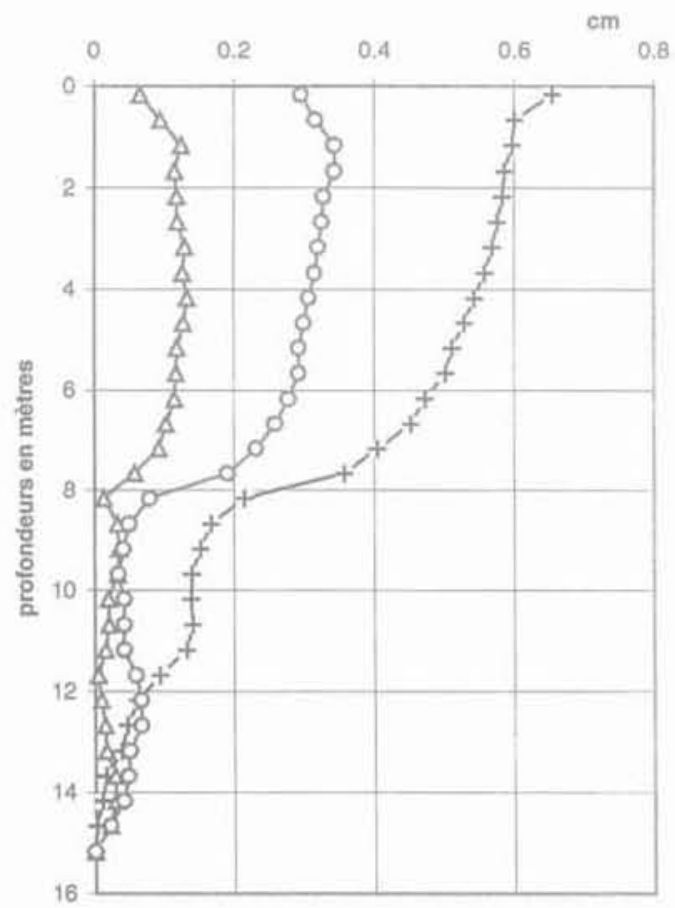

$+06 / 09 / 90-\Delta-06 / 03 / 90-0-08 / 06 / 90$

FG. 14 Rotations et déplacements cumulés mesurés par inclinométrie. Cumulated inclinometers measured displacements and rotations. 


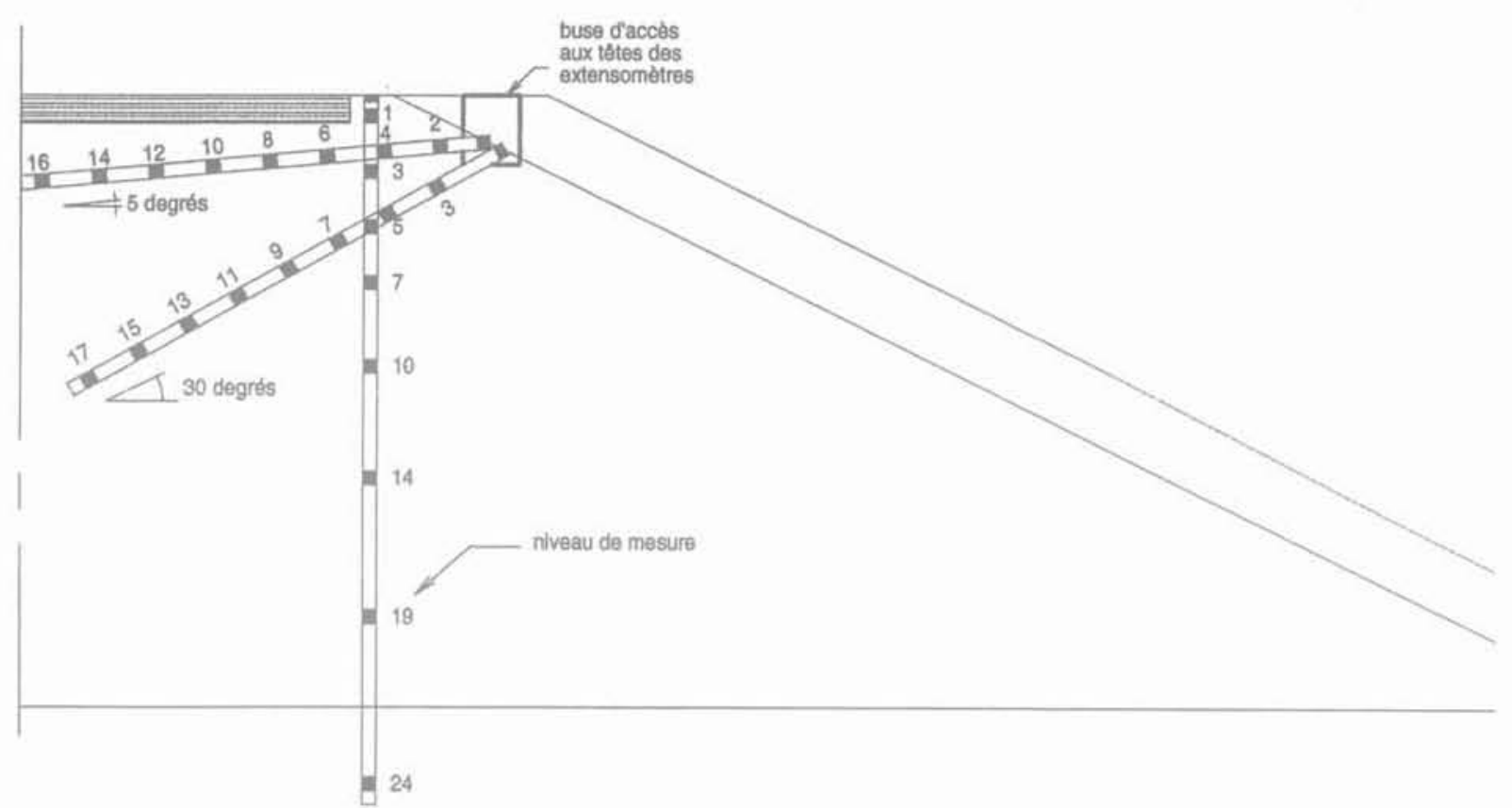

FiG. 15 Principe de confortation du remblai par élargissement de la plate-forme et épaulement du talus par un matériau traité. Reinforcement for widening of the platform.

La figure 14 donne une représentation graphique des rotations et des déplacements cumulés en fonction de la profondeur et du temps. Ces informations pouvaient faire craindre une évolution des déformations jusqu'à la rupture selon un plan correspondant à l'interface schématisée sur la figure 12. Une confortation a donc été réalisée, elle a consisté à mettre en œuvre un épaulement du remblai, jusqu'au niveau de la plate-forme, un élargissement de la voie étant prévu à moyen terme.

Les mesures extensométriques ont été réalisées avant et après réparation, ce qui donne une information intéressante sur l'efficacité de l'épaulement et sur le mécanisme des déformations. Les trois figures 16 à 18 donnent les résultats des mesures sous la forme de deux familles de graphes : la représentation de la déformation en fonction du temps, par rapport à un point fixe qui correspond à l'extrémité du tube (proche de l'axe pour les tubes inclinés) et les déformations cumulées en fonction de la profondeur à différentes dates caractéristiques.

Les extensomètres multipoints utilisés sont du type ( extensomètres de forage modèle WR-Flex $»$, La mesure des déformations relatives par rapport à la tête est faite au moyen d'une jauge de profondeur dont la résolution de la mesure est de l'ordre de $1 / 10$ de millimètre. Des capteurs électriques enregistrés de type rotatif ont été utilisés en début de période.

La figure 16 montre que l'épaulement a eu pour effet une accélération immédiate des vitesses de tassement, due à un accroissement de la charge. Les amplitudes à long terme ont été confirmées par nivellement.

Les mesures sur l'extensomètre incliné à 5 degrés montrent qu'avant travaux les principales extensions concernaient la tête et le repère placé à 2 mètres de distance. On observait en particulier un mouvement cyclique dont le minimum, dû au retrait, se situe en été. Après les travaux d'élargissement, le phénomène cyclique disparait, mais les extensions se poursuivent et intéressent des repères de plus en plus éloignés dans le corps du remblai.

Pour l'extensomètre incliné à 30 degrés (figure 18), on observe également, avant travaux, une extension pour les 3 mètres supérieurs et un mouvement cyclique perceptible jusqu'au repère situé à 3 mètres de distance de la tête. Le chargement dû à l'élargissement se traduit par une inversion des déformations avec une compression sensible jusquà 11 mètres de distance de la tête. Par contre, depuis 1996, les déformations se sont de nouveau inversées dans le sens des extensions.

Les principaux enseignements de cet ensemble de mesures sont les suivants:

- l'épaulement d'un talus par une surlargeur permet d'améliorer la stabilité générale, en particulier si les matériaux utilisés ont des caractéristiques mécaniques élevées, mais dans des proportions probablement assez faibles:

- la surcharge a une incidence évidente sur l’accélération des tassements ;

- la mise en place d'un matériau insensible à l'eau permet de réduire les cycles de gonflement et retrait et donc de supprimer le phénomène de reptation de pente ;

- le matériau mis en surlargeur, même s'il a des caractéristiques mécaniques exceptionnelles, ne permet pas de reprendre efficacement des efforts horizontaux ; les extensions actuellement observées indiquent donc que la reptation n'est probablement pas la seule cause des déformations horizontales et de la fissuration observée sur les chaussées.

Ce dernier point conduit à faire l'hypothèse que le corps d'un remblai peut se déformer par fluage, à un niveau donné, parce que les contraintes sont trop proches de l'état limite du matériau utilisé. Les mesures inclinométriques de la figure 7 ont été interprétées comme un phénomène de fluage confiné à la base du remblai (Fig. 8) et aucun calcul de stabilité ne permet- 


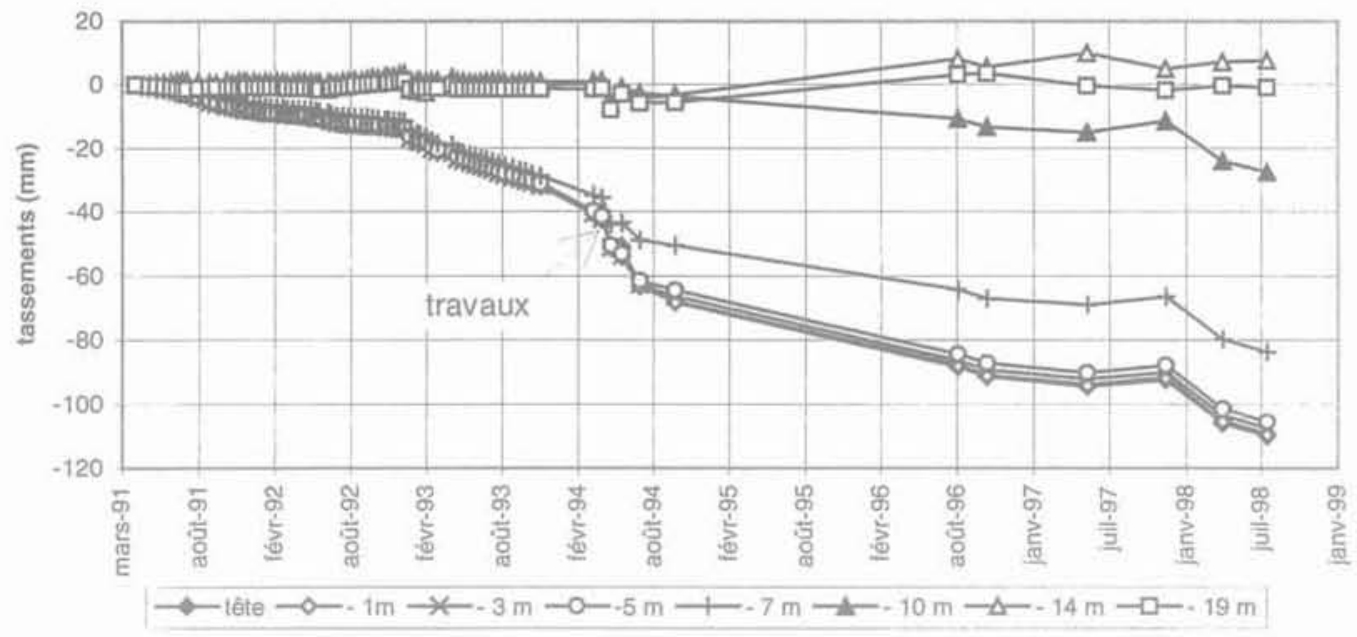

(a) - Tassements en fonction du temps par rapport au pied de tube à - 24

$\mathrm{m}$

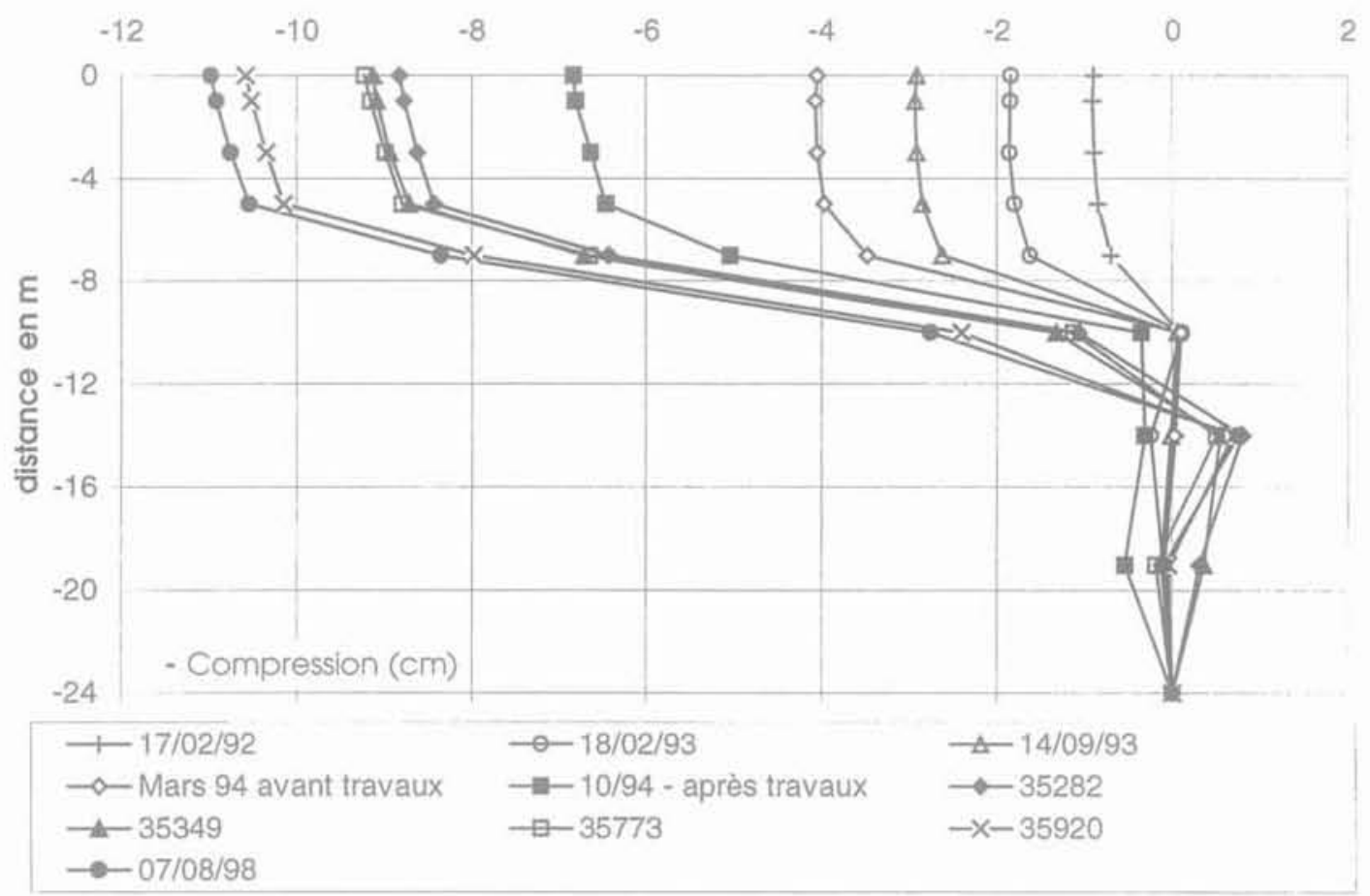

(b) - tassements cumulés par rapport au pied

FG is Tassements en fonction du temps et de la profondeur selon une verticale en crête de l'ancien talus. Settlement versus time and depth along the ancient slope crest.

trait de mettre en évidence un risque de rupture à ce niveau. Dans le cas de la figure 13, un schéma de rupture cornpatible avec les mesures inclinométriques nécessite de faire des hypothèses peu réalistes, à savoir par exemple un angle de frottement de $20^{\circ}$ et une fissure ouverte jusqu'à 8 mètres de profondeur, ou bien partiellement remplie d'eau. Le risque de rupture ne devait pas être exclu à cause des problèmes de sécurité mais, dans le cadre d'une réflexion sur le comportement réel du matériau, le phénomène de déformation par fluage confiné doit être également examiné.

Pour illustrer cette hypothèse, nous avons étudié les mesures faites sur un remblai routier de 13 mètres de haut, qui subit depuis sa mise en service une importante pathologie. Le corps du remblai est constitué d'un matériau argileux, parfois assez plastique, et les talus sont protégés par des matériaux insensibles à l'eau, argile traitée ou matériaux calcaires.

On a reporté sur la figure 19 les mesures de rotations et les déplacements horizontaux en crête de remblai et en regard, les tassements cumulés en fonction de la profondeur, mesurés avec un tassomètre multipoint placé dans l'axe de la voie, sur le même profil. Ces mesures mettent bien en évidence une déformation verticale et horizontale, au même niveau dans le corps du remblai, avec des amplitudes tout à fait similaires. Il ne s'agit donc pas de mouvements de type rotationnel avec une surface de rupture bien définie ; il n'y a d'ailleurs aucun désordre dans les talus alors que la plate-forme a subit d'importantes déformations, sous la forme de tassements et de fissures sans décalage vertical entre les lèvres. 


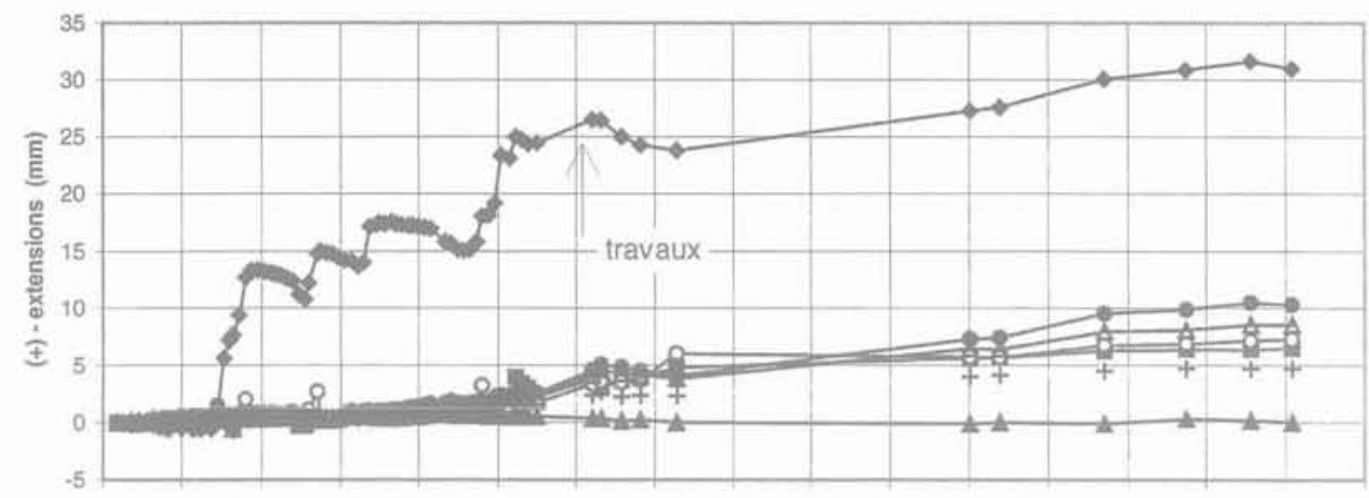

ma août- févr- aout- févr- aout- févr- août- févr- aout- févr- août- janv- juil- janv- juil- janv-

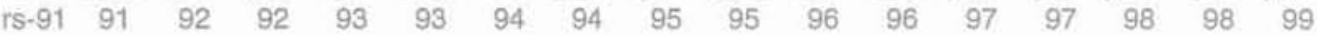

$\neg-14 m+-12 m-10-10 m \rightarrow 0-.8 m-\triangle-.6 m \rightarrow-4 m \rightarrow-2 m$

(a) - Déplacements par rapport à un repère à 16 mètres de la tête

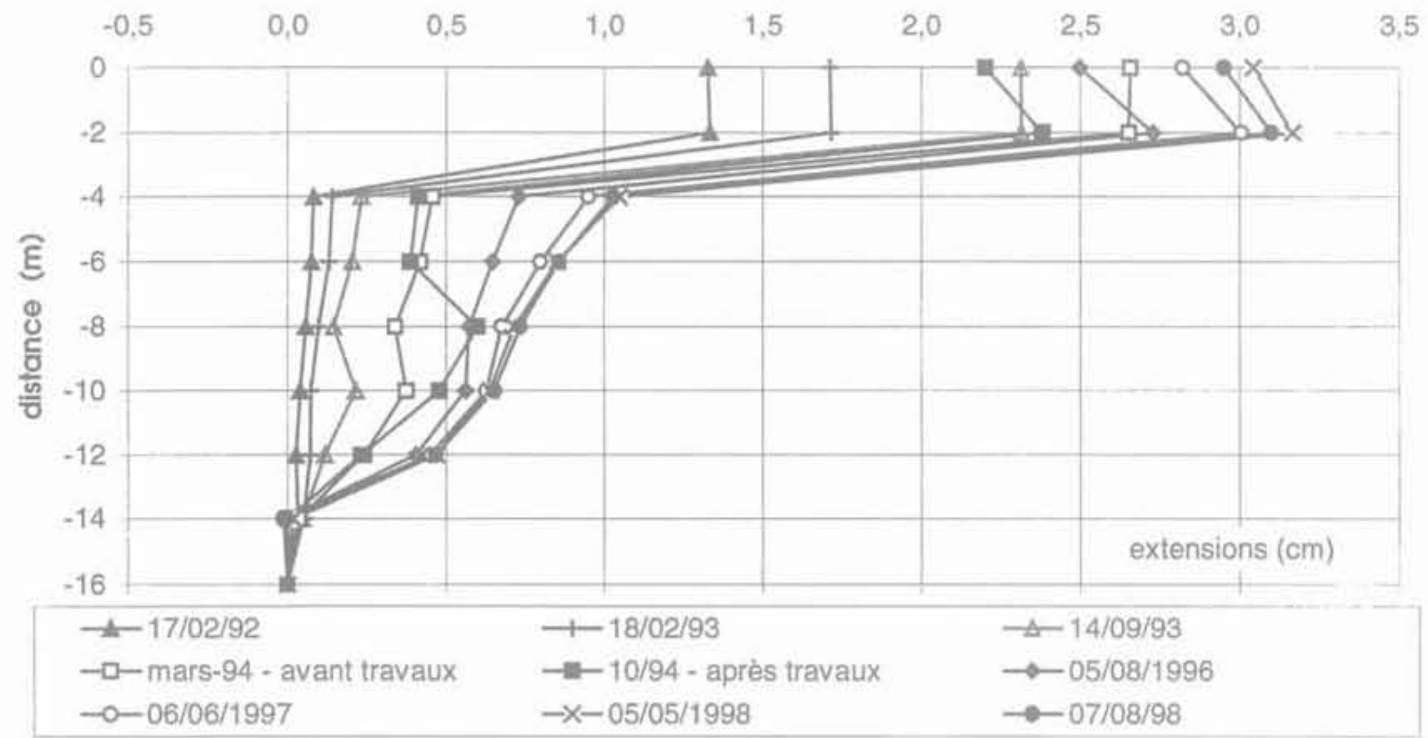

(b) - Extensions cumulées par rapport à l'ancrage

FG. 17 Déformations en fonction du temps et de la distance par rapport à un point fixe dans l'axe du remblai Extensomètre incliné à 5 degrés.

Deformations versus time and distance from a fixed point on the embankment axis.

Dans ce cas particulier, l'analyse des mouvements en fonction du temps met en évidence un amortissement, généralement représentatif d'un phénomène de fluage, ce qui n'est pas toujours vérifié, comme le souligne la figure 8.

\section{4}

\section{Conclusion}

Le premier enseignement que l'on peut déduire de cet ensemble de mesures est que le mécanisme des déformations des corps de remblai correspond probablement à la combinaison de plusieurs phénomènes physiques dont l'importance relative peut varier d'un ouvrage à l'autre. On ne donnera en conclusion qu'une liste, probablement incomplète, des principales causes.

Un certain nombre de causes sont bien connues et il serait dangereux de les oublier :
- le défaut de compactage et en particulier la mauvaise adaptation du niveau de compactage par rapport à la hauteur du remblai;

- le caractère évolutif du matériau, sous l'effet du compactage et surtout des variations de teneur en eau; ce point est particulièrement important si la mouture de la roche tendre initiale laisse un pourcentage important de vides ;

- les hétérogénéités et, en particulier, les contrastes de perméabilité qui favorisent localement les variations de teneur en eau.

D'autres causes sont moins souvent prises en compte :

- les risques de gonflement et retrait, qui concernent les sols gonflants mais aussi les argiles non saturées en général ; en effet, une variation de teneur en eau se traduit par une variation de succion, donc une déformation cyclique qui peut être à l'origine du phénomène de reptation de pente ; 


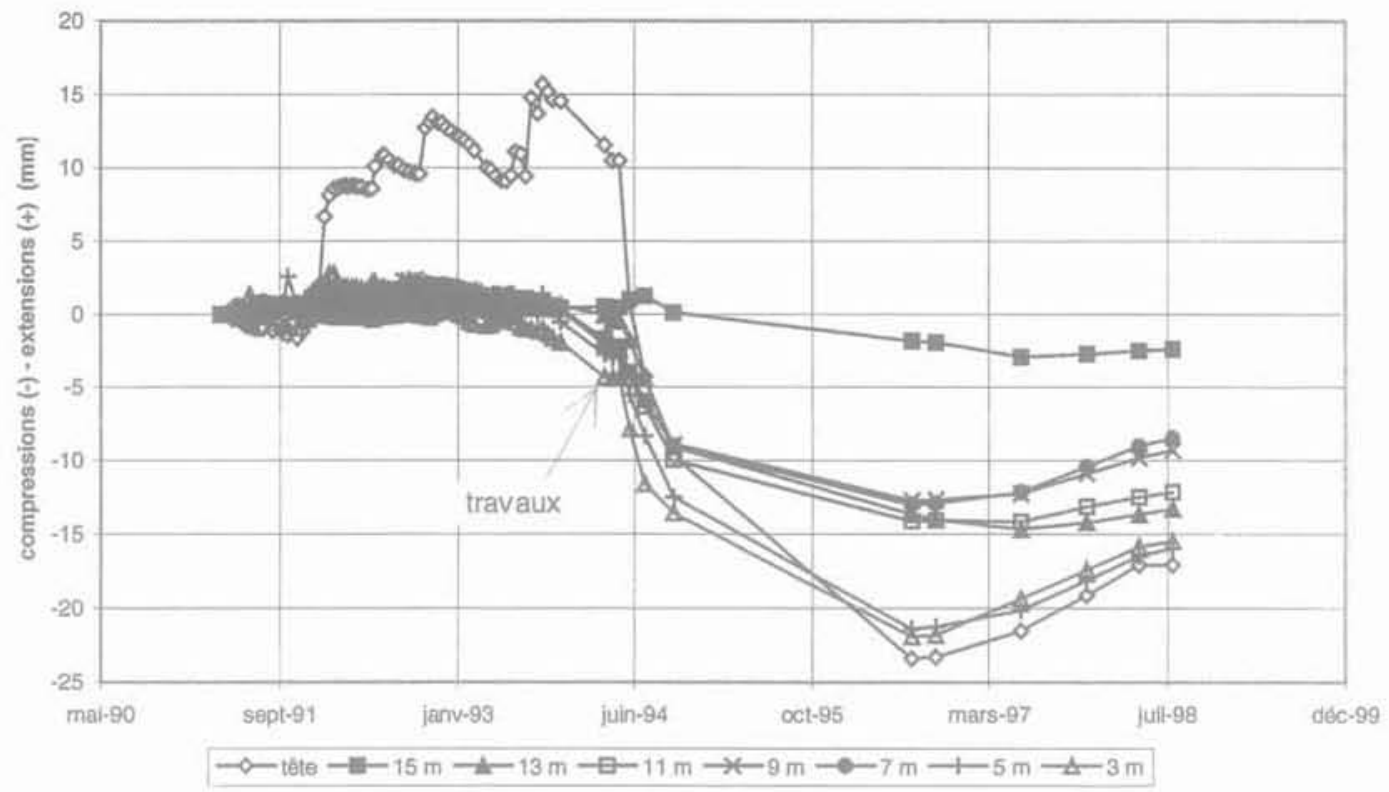

(a) - Déplacements par rapport à un repère à 17 mètres de la tête

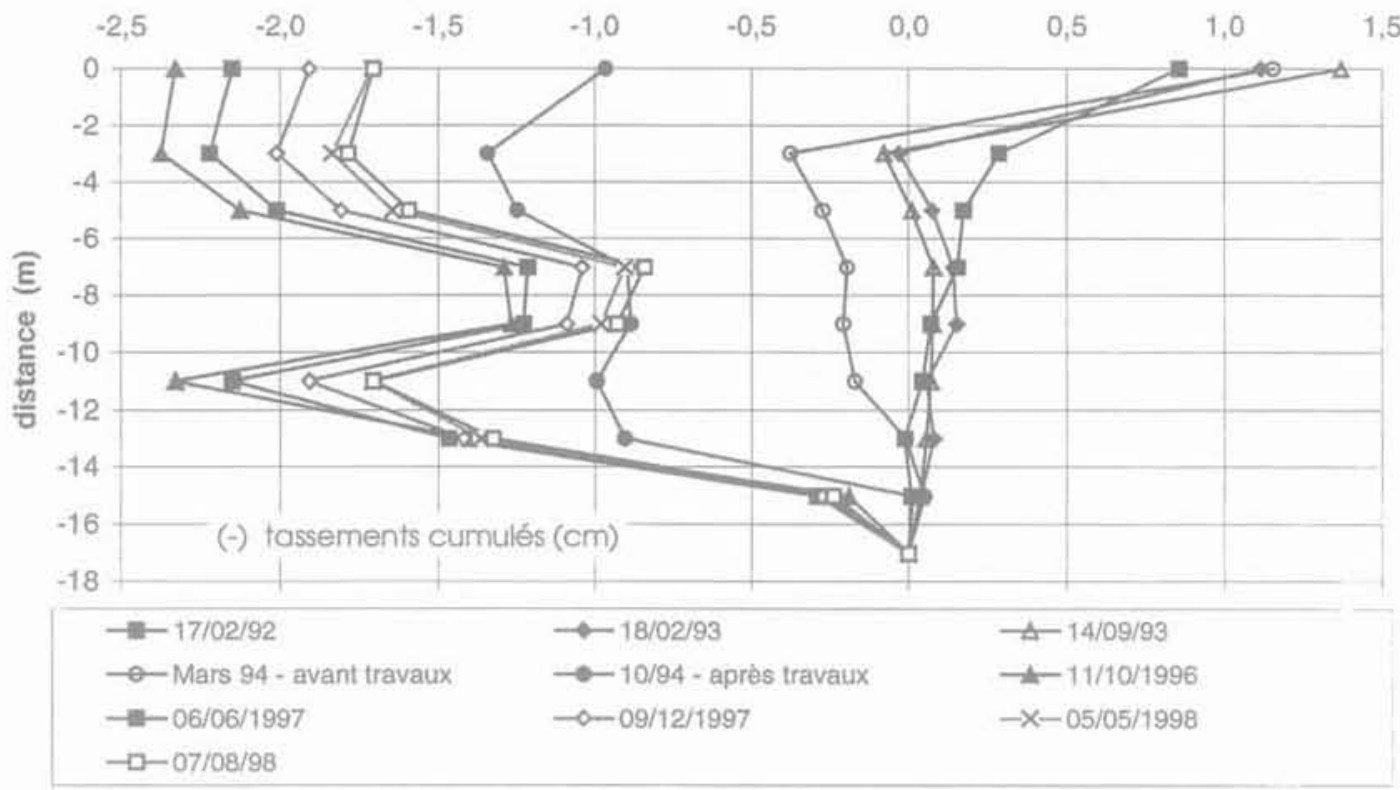

(b) - Déformations cumulés par rapport à l'ancrage à $17 \mathrm{~m}$

FGG 18 Déformations en fonction du temps et de la distance par rapport à un point fixe dans l'axe du remblai Extensomètre incliné à 30 degrés.

Deformations versus time and distance from a fixed point on the embankment axis.

- des déformations différées verticales et horizontales peuvent affecter certains matériaux compactés, soumis à un état de contrainte élevé ; le risque sera d'autant plus grand s'il se produit en plus des variations de teneur en eau à la base du remblai par remontées capillaires.

Ces réflexions montrent que les matériaux constitutifs de certains remblais doivent être étudiés non seulement pour définir les conditions de leur mise en œuvre et leur stabilité, mais aussi pour apprécier leur sensibilité aux changements de volume et leur déformabilité.

\section{REMERCIEMENTS}

L'auteur de cet article tient à remercier la sociêté des Autoroutes du Sud de la France et, plus particulièrement, les responsables de la DRE d'Agen pour la confiance qu'ils nous ont témoignée en nous permettant d'instrumenter et de suivre de nombreux remblais dans un cadre expérimental mais aussi pour la surveillance d'ouvrages courants. 

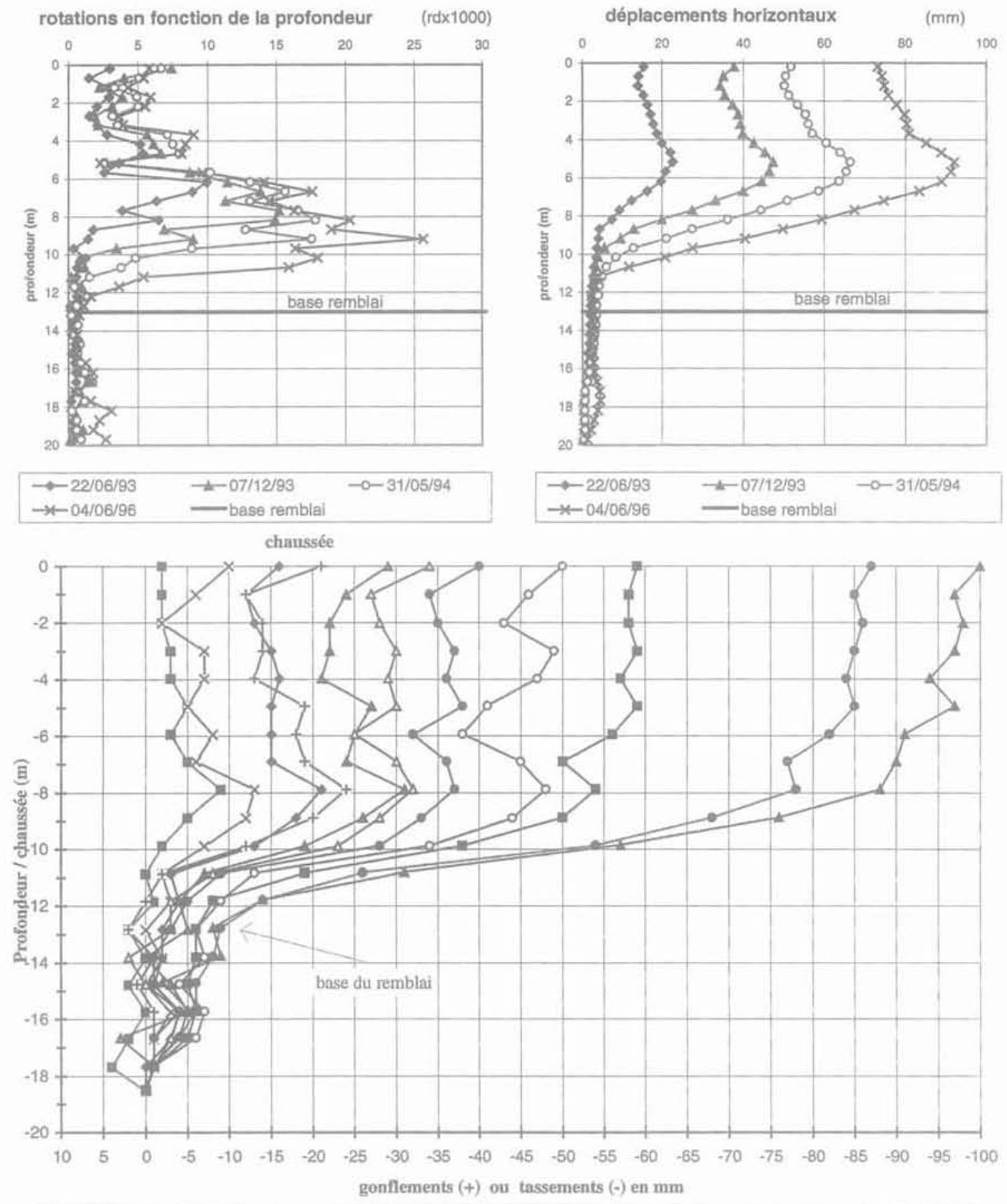

\begin{tabular}{|c|c|c|c|c|c|}
\hline$=-31-$ mars -93 & $-x-05-m a i-93$ & $\leadsto-22$-juin-93 & $+27-j u i l-93$ & $-4-23$-sept-93 & $-d-07$-déc-93 \\
\hline$\rightarrow-22$-févr-94 & $-\infty-31-m a i-94$ & $-m-07$-oct-94 & $\rightarrow-03-$ oct-95 & - -04-jun-96 & \\
\hline
\end{tabular}

FG.19 Comparaison des déplacements horizontaux en crête de talus et des tassements dans l'axe d'un remblai argileux de 13 mètres de haut.

Horizontal displacements at the slope crest versus settlement on the axis of a 13 meters high clay made embankment.

\section{Bibliographie}

Thouret D. Puech J-P. Mieussens C - "Autoroute A62. Suivi d'un grand remblai en marne: bilan de dix années d'observation 1 . Revue générale des routes et autoroutes, $n^{\circ} 700$, octobre 1992, p. 23-27.

Mieussens C.. Camapum de Carvalho J. - Remblais en marne Désordre, étude, confortation. Document technique édité par
Scetauroute et le Laboratoire central des ponts et chaussées, mars 1986.

Mieussens C. - $\alpha$ Les remblais en marne - Etudes, pathologie et techniques de réparation $n$. Texte de la conférence prononcée aux journées du $70^{\circ}$ anniversaire de la Junta Autonoma de Estradas, Lisbonne, septembre 1997. 